\title{
Effectiveness and Safety of Low-Threshold Opioid-Agonist Treatment in Hard-To-Reach Populations with Opioid Dependence
}

\author{
Fatemeh Chalabianloo ${ }^{a, b}$ Christian Ohldieck ${ }^{a}$ Øystein A. Haaland ${ }^{b}$ \\ Lars Thore Fadnes $^{a, b}$ Kjell Arne Johansson ${ }^{a, b}$

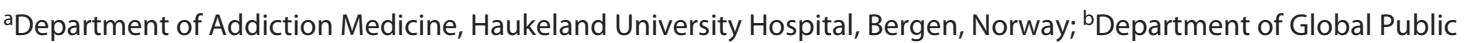 \\ Health and Primary Care, University of Bergen, Bergen, Norway
}

\section{Keywords}

Low-threshold · opioid-agonist treatment · Hard-to-reach populations · Retention - Death · Overdose · Healthcare utilization · Access · Delivery platform

\begin{abstract}
Objectives: Opioid-use disorder is related to premature death worldwide. Opioid-agonist treatment (OAT) is an effective treatment for opioid dependence. OAT delivery platforms may influence treatment access and outcomes, especially for the most vulnerable groups. The aim of this study was to determine the effectiveness and safety of low-threshold OAT compared to the standard treatment. Methods: Patients with diagnosed opioid dependence undergoing lowthreshold OAT at the Bergen delivery platform in Norway were enrolled in a cohort study in 2014-2019. A national OAT cohort was the reference group. The main outcomes were treatment retention, the use of illicit opioids, non-fatal overdose, overdose death, and all-cause mortality during the first year following treatment initiation and the full treatment period. Additionally, healthcare utilization in the periods before and during OAT was investigated. Results: Compared to the reference cohort, the low-threshold cohort $(n=128$, mean age: 38 years, women: $28 \%$ ) showed treatment retention rates of $95 \%$ versus $92 \%$, illicit opioid use of $7 \%$ versus $10 \%$, non-fatal overdose of $7 \%$ versus $6 \%$, and death at $1.0 \%$
\end{abstract}

karger@karger.com www.karger.com/ear

Karger $\frac{1}{6}$
C 2021 The Author(s).

Published by S. Karger AG, Basel

This is an Open Access article licensed under the Creative Commons Attribution-NonCommercial-4.0 International License (CC BY-NC) (http://www.karger.com/Services/OpenAccessLicense), applicable to the online version of the article only. Usage and distribution for commercial purposes requires written permission. versus $1.3 \%$, respectively. The incident rate ratios (IRRs) for healthcare utilization increased substantially during the OAT period compared to the period before; the IRR increased by 3.3 (95\% confidence interval (Cl): $2.8,3.9)$ and 3.4 (95\% Cl: 3.1 , 3.9) for all in- and outpatient healthcare, respectively. Conclusions: Low-threshold OAT was at least as effective and safe as the standard OAT in terms of treatment retention, the use of illicit opioids, non-fatal overdose, and death. Healthcare utilization increased during the OAT compared to the period before. Lowering the threshold for OAT entrance within proper delivery platforms should be broadly considered to reduce harm and improve healthcare access among patients with opioid dependence.

(c) 2021 The Author(s)

Published by S. Karger AG, Basel

\section{Introduction}

Opioid-use disorder is a major contributor to premature death worldwide $[1,2]$. In 2019, around 46,000 deaths (5\% of all deaths) and 4,700,000 disability-adjusted life years ( $6 \%$ of total disease burden) were caused by opioid-use disorders in the USA among people under the age of 70 years [3]. Norway is one of the European countries with a high prevalence of drug-related deaths of which about $90 \%$ are caused by opioid-related overdoses [4]. The frequency of drug-induced deaths was 6.1 per 
100,000 people of $15-64$ years of age in 2020 , and these were mostly related to the use of heroin, morphine, and other synthetic/semi-synthetic opioids that were either prescribed or illicitly acquired [5]. This is a worrisome trend. Opioid-agonist treatment (OAT) with methadone (Met) and buprenorphine (Bup) is an effective treatment for opioid dependence that improves survival, abstinence from illicit opioids, and quality of life [6-11]. However, barriers exist with respect to access to OAT, and the optimal clinical setting is unclear [12]. Understanding how OAT delivery platforms may influence treatment access and outcomes is important, especially for populations that are hard to reach through ordinary care systems [13].

Access to OAT and treatment outcomes may be limited as a result of long waiting lists, strict eligibility criteria, and prerequisites such as abstinence from illicit substances before treatment initiation and a lack of treatment individualization. Reasonably, decreasing the waiting times and removing treatment initiation barriers are crucial to increasing the coverage and effectiveness of OAT and reducing opioid-related deaths $[8,14]$. Low-threshold services provide voluntary-based interventions without requiring abstinence from illicit substances as a prerequisite to entering treatment [15]. The inappropriate selection and doses of OAT medications have also been shown to be associated with an increased risk of dropout and mortality and are considered barriers to OAT effectiveness $[9,13]$. Bup seems safer than Met with a potentially lower risk of overdose, but it is less effective in retaining patients in treatment and may aggravate heroin withdrawal symptoms in the induction phase [8]. Access to OAT has also been shown to be associated with a shortened time to Met initiation [16]. Sufficiently high Met or Bup doses are crucial for treatment retention [17-19] and optimal attendance in care [20]. However, Met enhances the risk of death in the first 4 weeks during the induction phase, especially when combined with other CNS suppressants $[8,9,21-23]$. Nevertheless, there is strong evidence of a significantly higher risk of all-cause mortality without OAT than with OAT $[8,9,22-25]$. The optimal use of low-threshold services represents a trade-off between safety at treatment initiation and access to care; however, better evidence on the safety and effectiveness of low-threshold OAT is needed.

Some countries have shown promising results with respect to improved treatment retention and reduced morbidity and mortality in municipal low-threshold OAT settings [18, 20, 26-30]. Despite this, low-threshold OAT has not been investigated in depth as part of a specialized healthcare system. In Norway, few low-threshold OAT options are available as part of municipal care plans that encompass harm reduction approaches [31], and lowthreshold OAT has not been established as a widely available approach in specialized healthcare services [32]. To include more vulnerable groups of people with opioid dependence who do not reach ordinary OAT care, the Department of Addiction Medicine at Haukeland University Hospital in Bergen, Norway, established a low-threshold clinic with drop-in assessments to determine OAT eligibility without the need for a referral or abstinence from illicit substance use. Both Bup and Met are offered daily in an integrated outpatient setting in Bergen (the Bergen OAT [B-OAT] delivery platform) with close clinical observation and other interdisciplinary approaches, which is in line with the treatment offered by ordinary OAT clinics.

In this study, we primarily assessed the effectiveness (treatment retention and the use of illicit opioids) and safety (non-fatal overdose, overdose death, and all-cause mortality) of low-threshold OAT in the B-OAT compared to the standard OAT in Norway. We also aimed to determine the characteristics of the study participants and to investigate changes in their use of healthcare services before and during low-threshold OAT.

\section{Methods}

\section{Study Population}

Since the establishment of the low-threshold outpatient OAT clinic as part of the B-OAT in November 2014, the centre has admitted people with substance-use disorders who have walked into the clinic voluntarily. Retrospectively, 128 of these patients were eligible for inclusion in the present study. The participants were diagnosed with opioid dependence in accordance with the relevant International Classification of Diseases-10 criteria and enrolled in the low-threshold OAT outpatient clinic at the Department of Addiction Medicine, Haukeland University Hospital, in Bergen from November 2014 to December 2019 (i.e., the lowthreshold cohort [LTC]). The department is responsible for the treatment and follow-up of $>1,000$ patients undergoing OAT through ordinary or low-threshold admission using a well-established B-OAT delivery platform. The treatment is integrated with psychosocial approaches provided by interdisciplinary teams, which include addiction specialists, nurses, social workers, and psychologists. Irrespective of whether they are followed up at the low-threshold or an ordinary OAT clinic, patients receive medication mainly by direct observation at the clinic, except for the most stabilized patients who receive medication and are observed at a pharmacy. The take-home doses are based on individual assessments. Other medical interventions, such as treatment for hepatitis $\mathrm{C}$ virus infection, are also provided at OAT clinics [33]. The national OAT cohort in Norway (national reference cohort [NRC]), which comprises 7,900 patients and includes different 
delivery platforms due to the diverse geographical and organizational conditions, was used as the reference group, not as a control group [34]. Most of the NRC patients had been undergoing OAT for several years as this was established in Norway in the late 1990s [34].

The following data on the LTC were obtained retrospectively from the electronic patient journal system for the study period: patient age and gender; type and dose of OAT medication; the use of illicit substances and alcohol (based on self-reported data and urine screening tests); drop out from and readmission to OAT; social status; physical and mental health status; in- or outpatient admissions due to acute and non-acute somatic, psychiatric, or substance-related incidents before and during OAT; and death during the treatment period, including the cause of death established via autopsy. For the NRC, the individual patient data were unavailable, so aggregated data from the last annual national report [34] were used as reference data.

\section{Low-Threshold Settings}

Eligibility criteria based on the national OAT guidelines [32] were applied for inclusion in OAT for both the LTC and NRC. The differences related to the prerequisites for treatment entrance and initiation and the OAT delivery platform. A low-threshold intervention was defined as outpatient initiation with Met or Bup and without a prerequisite referral or abstinence from substance use (no in-bed detoxification or urine screening was required). A standard intervention was defined as treatment initiation using the same medications but with the need for a referral and abstinence from substance use, which was confirmed using urine tests showing no illicit substances other than the prescribed opioid initiated as a stabilizing medication prior to dose upscaling to reduce the risk of overdose. The standard routine was inpatient detoxification before starting Met treatment. In certain circumstances, Bup can be started on an outpatient basis, but the same requirements apply as for Met.

\section{Study Outcomes}

The primary outcomes were treatment retention, the use of illicit opioids, non-fatal overdoses, overdose deaths, and all-cause mortality, both during the first year following treatment initiation and during the full treatment period. The cumulative findings were divided by the mean duration of OAT, which was 4 years, to present the yearly rate as the reports from the national reference OAT cohort reflect annual data. The other outcomes were contact with healthcare services for acute and non-acute somatic, psychiatric, and substance-related incidents in the years before OAT (from January 2007 to OAT initiation in the LTC; mean duration: 9 years, range: $7-12$ years) and during OAT (from treatment entry until December 2019 or treatment termination/death; mean duration: 4 years, range: $1-5$ years).

The treatment initiation period was considered the period of dose escalation and titration until the target OAT medication dose was achieved, while the stabilization period was considered the period starting 2 weeks after the target dose had been achieved. Box 1 presents the applied procedures at the low-threshold OAT clinic. A healthcare utilization incident was defined as an acute, subacute, or non-acute health complaint resulting in contact with the healthcare system and clustered into one of the somatic, psychiatric, or substance-related subgroups based on the primary International Classification of Diseases-10 condition for each contact.

Low-Threshold Opioid-Agonist

Treatment
Box 1. Dose titration of methadone and buprenorphine at lowthreshold OAT clinic

\begin{tabular}{|c|c|c|c|c|c|}
\hline \multicolumn{3}{|c|}{ Methadone } & \multicolumn{3}{|c|}{ Buprenorphine } \\
\hline days & $\begin{array}{l}\text { dose, } \\
\mathrm{mg}\end{array}$ & $\begin{array}{l}\text { observation, } \\
\text { h }\end{array}$ & days & $\begin{array}{l}\text { dose, } \\
\mathrm{mg}\end{array}$ & $\begin{array}{l}\text { observation, } \\
\text { h }\end{array}$ \\
\hline $1-3$ & 30 & 2 & 1 & $4+4$ & $0.5+1$ \\
\hline $4-6$ & 40 & 2 & 2 & 8 & 1 \\
\hline $7-9$ & 50 & 2 & 3 & 12 & 1 \\
\hline $10-12$ & 60 & 2 & 4 & 16 & 1 \\
\hline $13-15$ & 70 & 1 & & & \\
\hline $16-22$ & 80 & 1 & & & \\
\hline $23+$ & 90 & 1 (the first week) & & & \\
\hline
\end{tabular}

\section{Statistical Analysis}

Stata/SE version 16.0 (Stata Corp, College Station, TX, USA) was used for statistical analyses. The basic descriptive analyses were presented as means with standard deviations and ranges for the continuous variables. The incident rate ratios (IRRs) and differences between the incidents leading to healthcare utilization during treatment and in the period before OAT were calculated using the Poisson regression test. All the calculations were adjusted for time in the Poisson regression analyses. The exact $p$ values with $95 \%$ confidence intervals (CIs) were reported, and values below 0.05 were considered statistically significant.

\section{Results}

The clinical and sociodemographic characteristics of the LTC at OAT entrance are presented in Table 1. As a supplement, a typical patient at the low-threshold clinic is described qualitatively in Box 2. For the NRC, not all the corresponding data at OAT entrance were available, but some data from the full treatment period were accessible and were thus included. Overall, 128 LTC patients were included in this study. The LTC had a mean age of 38 years, and 36 (28\%) were women (the remaining patients were men) versus 46 years and $31 \%$, respectively, for the 7,900 NRC patients at the end of 2019. In the LTC, $98 \%$ were without regular jobs and living on social and disability benefits, $68 \%$ had an education level below secondary school, $74 \%$ were single, and $96 \%$ had not cared for children. Furthermore, 68 (53\%) patients lacked permanent residence status, of which $22(32 \%)$ were living in family's or friends' homes, 19 (28\%) in shelters/care homes, and 27 (40\%) were homeless. Among them, 121 (95\%) patients used other substances in addition to illicit opioids, and the same number had been injecting substances at treatment entrance. Ninety-five patients (74\%) entered OAT for the first time at the low-threshold clinic, 
Box 2. A typical patient at the low-threshold OAT clinic

John is a single man in his thirties. He injects heroin daily and also uses multiple other substances regularly. He has an untreated hepatitis $\mathrm{C}$ virus (HCV) infection and has suffered from generalized anxiety since he was a young boy, which made him drop out of secondary school. He isolates himself socially and has had limited contact with the healthcare system, social services, or even his family. He usually spends the night in night-homes, shelters, or sometimes with the random friends who share drugs with him. He receives social security benefits as the only formal income using it mainly to finance heroin and other drugs, if any. During the last months, he has been admitted to the emergency unit several times due to intoxication with heroin combined with other drugs. He has not tried OAT before, despite thinking about it as a treatment approach for a longer period. He has not been able to attend healthcare appointments. During the last episode of heroin intoxication, the doctor informed him about the possibility of showing up at the low-threshold clinic without a referral or making an appointment in advance. This information was given while he had to hurry out of the emergency unit having an irresistible craving to shoot heroin. After a couple of tough weeks since his previous discharge from the emergency ward, he decided to contact the low-threshold clinic hoping to start OAT as soon as possible. Two weeks after his initial drop-in visit, he had initiated OAT with methadone reaching $90 \mathrm{mg}$ per day. He was stabilized during the first month, and one year later, he still received OAT. The multidisciplinary team helped him reach diverse health and social services. John received an apartment, daily income, health examinations including testing and treatment for $\mathrm{HCV}$, and psychological consultations relating to handling of anxiety, along with some offers on physical activities and voluntary job projects. He had a couple of admissions in the emergency substance unit during the first year after commencing OAT due to intoxication with drugs other than heroin. Since initiation of OAT, he has been followed by daily observed intake of medications and regular consultations at the clinic. Now, he has a more stable life situation and stopped injecting heroin and other substances, although, he still uses some illicit benzodiazepines orally and smokes cannabis regularly. He has also completed HCV treatment which further motivates him to avoid injecting drugs. The next step is to further establish purposeful daily activities as part of his recovery process.

while the remainder were recorded as restarting OAT after having been out of standard treatment for varying lengths of time.

The OAT medication at treatment initiation was Met in $34 \%$ of the patients or Bup in $66 \%$, with a mean daily dose of 91 and $16 \mathrm{mg}$, respectively. Seven patients in each medication group changed to the other group during the treatment period. The mean time from contact with the clinic to medication initiation was 14 days, and the mean duration of time to achieve the target dose and stabilization was 25 days. The average time for OAT was 4 years.
One-quarter of the patients had had very limited or no contact with healthcare or social services before OAT entrance, although 83 (65\%) and 118 (92\%) patients were suffering from various chronic somatic and/or psychiatric conditions, respectively.

Table 2 provides a summary of the primary outcomes during the first treatment year and the cumulative data during the full study period for the LTC, which are shown as the total data and the data after being sorted by OAT medication. The corresponding cumulative data for the NRC for the full treatment period are provided as the reference data. The cumulative retention rate in treatment for the LTC was $95 \%$, with 26 patients terminating OAT at different times across the full treatment period. Twenty-one $(81 \%)$ of these patients ceased treatment voluntarily, of which 6 ceased treatment following planned tapering, and 15 were no-shows at the clinic for a period exceeding 3 months. During the first treatment year, 20 patients dropped out, of which 9 re-entered OAT before the end of this period. Thus, 11 terminated OAT after repeated dropouts, and 1 patient died during this period. Five patients died during the full study period (the cumulative rate of all-cause mortality was 1.0\%), 4 in the Bup group after the first treatment year, and 1 in the Met group during the induction period. Only one death in the Bup group was attributed to somatic causes, whereas drug-related causes were possible explanations in the remaining 4 cases. The autopsy reports and post-mortem findings based on forensic toxicological tests indicated diverse substance use, typically benzodiazepines (and heroin in 1 case), in addition to OAT medication, although other possible causalities could not be ruled out.

Regarding the use of illicit opioids, 37 (7\%) patients had used heroin or other opioids during the last treatment month. Thirty-five patients (10 in the Met and 25 in the Bup group) had at least one recorded non-fatal overdose during the full treatment period, giving a cumulative rate of $7 \%$ (6\% and $7 \%$ in each respective group) for non-fatal overdoses. Thirty-two ( 9 and 23 patients in each respective group) of the 35 patients experienced at least one non-fatal overdose within the first year, of which 10 dropped out repeatedly following OAT initiation, but no patient died during this period. Five of the 32 patients terminated OAT during the first treatment year. Finally, 12 of the 35 patients terminated OAT during the full treatment period, 3 (all in the Bup group) due to drugrelated death (Table 3 ).

Table 4 shows the utilization of in- and outpatient healthcare services for acute and non-acute somatic, psychiatric, or substance-related incidents before and during 
Table 1. Baseline characteristics of the participants in a study of effectiveness and safety of low-threshold OAT

\begin{tabular}{|c|c|c|}
\hline Gender, female, $n(\%)$ & $36(28)$ & $(31)$ \\
\hline \multicolumn{3}{|l|}{ Living status, $n(\%)$} \\
\hline Residence (none or temporary) & $68(53)$ & $(20)$ \\
\hline Income (no regular job) & $126(98)$ & $(82)$ \\
\hline Care for children (no care) & $123(96)$ & No data \\
\hline Substance use in the last month before OAT initiation, $n(\%)$ & $121(95)$ & \\
\hline Heroin/other opioids (dominantly used) & $67(52) / 61(48)$ & $(10)$ \\
\hline Amphetamines & $83(65)$ & $(15)$ \\
\hline Benzodiazepines (illicit/prescribed) & $118(92)$ & (39) \\
\hline \multicolumn{3}{|l|}{ OAT medication at treatment start, $n(\%)$} \\
\hline Met & $44(34)$ & $(37)$ \\
\hline Changed to Bup latter & $7(16)$ & No data \\
\hline Bup & $84(66)$ & $(59)$ \\
\hline Changed to Met latter & $7(8)$ & No data \\
\hline \multicolumn{3}{|l|}{ Maintenance dose of OAT, mean (SD; range), mg } \\
\hline Met & $91(21 ; 30-150)$ & 91 \\
\hline Bup & $16(5 ; 2-26)$ & 15 \\
\hline Time from the first contact to treatment start, mean (SD; range), days & $14(19 ; 0-158)$ & No data \\
\hline Time to dose stabilization after starting OAT, mean (SD; range), days & $25(16 ; 15-120)$ & No data \\
\hline Duration of OAT, mean (range), years & $4(1-5)$ & $10(1-20)$ \\
\hline No/limited contact with health or social services, $n(\%)$ & $32(25)$ & No data \\
\hline Attention deficit disorders & $32(27)$ & No data \\
\hline Other chronic mental diseases & $21(18)$ & No data \\
\hline
\end{tabular}

OAT, opioid-agonist treatment; HCV, hepatitis C virus; COPD, chronic obstructive pulmonary disease; SD, standard deviation; Met, methadone; Bup, buprenorphine. *Only aggregated proportions (no numbers) and means (no ranges) were available for some indicators (no all), during the entire treatment period in OAT (no data were available at entrance OAT). In total, $82 \%$ out of 7,900 patients were interviewed in the survey, and 7-22\% of questions were answered as "not known," giving about 25-40\% missing data in the last national annual survey [34]. This should be considered when interpreting the data.

OAT. Overall, 58,322 contacts were recorded for the 128 patients in the LTC, of which 46,296 were related to the observed intake of OAT medication and follow-ups during the same course of treatment. These were excluded from the analyses to avoid introducing bias. The remaining 12,025 contacts were related to other healthcare contacts during the same period. In almost all cases, the use of healthcare services increased significantly during the
OAT period compared to the period before treatment. The IRRs were 3.3 (95\% CI: $2.8,3.9)$ and 3.4 (95\% CI: 3.1, 3.9) for all the in- and outpatient healthcare, respectively. However, the IRR did not increase significantly for the use of inpatient care for acute psychosis, depressive episodes, acute psychiatric incidents, and acute infections or for outpatient care for acute depression and other acute psychiatric and cardiovascular incidents. 
Table 2. Primary outcomes in a study of effectiveness and safety of low-threshold OAT

\begin{tabular}{|c|c|c|c|c|c|c|c|}
\hline \multirow{2}{*}{ Primary outcomes } & \multicolumn{3}{|l|}{ first year } & \multicolumn{3}{|c|}{ cumulative } & \multirow{2}{*}{$\begin{array}{l}\text { National reference OAT } \\
\text { cohort }(n=7,900), n(\%) \\
\text { annual } \\
\text { total }\end{array}$} \\
\hline & total & Met & Bup & total & Met & Bup & \\
\hline Retention in OAT & $116(91)$ & $40(91)$ & $76(90)$ & $102(95)$ & $37(96)$ & $65(94)$ & $7,233(92)$ \\
\hline Planned tapering & 0 & 0 & 0 & 6 & 2 & 4 & $203(40)$ \\
\hline Dropout $^{c}$ & 11 & 3 & 8 & 15 & 4 & 11 & $307(60)$ \\
\hline Involuntarily terminated & $0(0)$ & $0(0)$ & $0(0)$ & $0(0)$ & $0(0)$ & $0(0)$ & $44(7)$ \\
\hline Death & $1(8)$ & $1(25)$ & $0(0)$ & $5(19)$ & $1(14)$ & $4(21)$ & $112(17)$ \\
\hline Illicit opioid use in the last month & n.a. & n.a. & n.a & $37(7)^{a}$ & $22(9)^{\mathrm{a}}$ & $31(7)^{\mathrm{a}}$ & $790(10)$ \\
\hline Heroin & - & - & - & $31(6)^{a}$ & $12(7)^{\mathrm{a}}$ & $19(6)^{\mathrm{a}}$ & No data \\
\hline Somatic causes & $0(0)$ & $0(0)$ & $0(0)$ & $1(20)$ & $0(0)$ & $1(25)$ & $62(72)$ \\
\hline Violence & $0(0)$ & $0(0)$ & $0(0)$ & $0(0)$ & $0(0)$ & $0(0)$ & $10(12)$ \\
\hline
\end{tabular}

Bup, buprenorphine; Met, methadone; $\mathrm{Cl}$, confidence interval; OAT, opioid-agonist treatment; n.a., not applicable due to too few data; NRC, national reference cohort; LTC, low-threshold cohort. ${ }^{a}$ The per cent is divided by the mean duration of OAT, which is 4 years, to present the yearly rate. The numbers for the national reference OAT cohort present annual data (the last reporting year is 2019 [34]). ${ }^{b}$ Eight percentage in 2019 and 7\%: cumulative from 2013 to 2019 in the national cohort. No Cls are available in the annual reports. ${ }^{\mathrm{C}}$ In total, 20 patients dropped out from medication during the first year after treatment start; however, 9 of those restarted OAT within the same period ( 6 at low-threshold outpatient settings and 3 at inpatient settings), then the remaining 11 were out of the treatment at the end of the first treatment year. ${ }^{\mathrm{d}}$ All the death causes were based on autopsy reports in the LTC, but only $6 \%$ of the death causes in the reference cohort were suggested based on autopsy reports and the remaining were based on assumptions. ${ }^{\mathrm{e}}$ Fifty-three percentage of those who died in the NRC used Met and $41 \%$ used Bup, while under active medication. The remaining is not presented in the annual report due to uncertain data.

Table 3. Participants with non-fatal overdoses during the treatment period in a study of effectiveness and safety of low-threshold OAT

\begin{tabular}{llll}
\hline & Total, $n=35$ & Met, $n=10$ & Bup, $n=25$ \\
\hline Gender, female/male, $n / N$ & $5 / 30$ & $1 / 10$ & $4 / 20$ \\
Age, mean (SD; range), years & $33(9 ; 19-51)$ & $32(8 ; 22-47)$ & $34(9 ; 19-51)$ \\
Overdoses occurred during the first treatment year & 32 & 9 & 23 \\
Repeated dropouts during the first treatment year & 10 & 3 & 7 \\
Terminated due to no show* during the first treatment year & 5 & 2 & 3 \\
Died during the first treatment year & 0 & 0 & 10 \\
Terminated during the entire treatment period & 12 & 2 & 5 \\
$\quad$ Causes of OAT termination & 7 & 2 & 2 \\
$\quad$ No show* at OAT clinic & 2 & 0 & 3 \\
$\quad$ Planned tapering & 3 & 0 & 3 \\
\hline
\end{tabular}

Bup, buprenorphine; Met, methadone; OAT, opioid-agonist treatment; SD, standard deviation. * At least 4 consecutive absences with no return to OAT clinic at the following 3 months. 


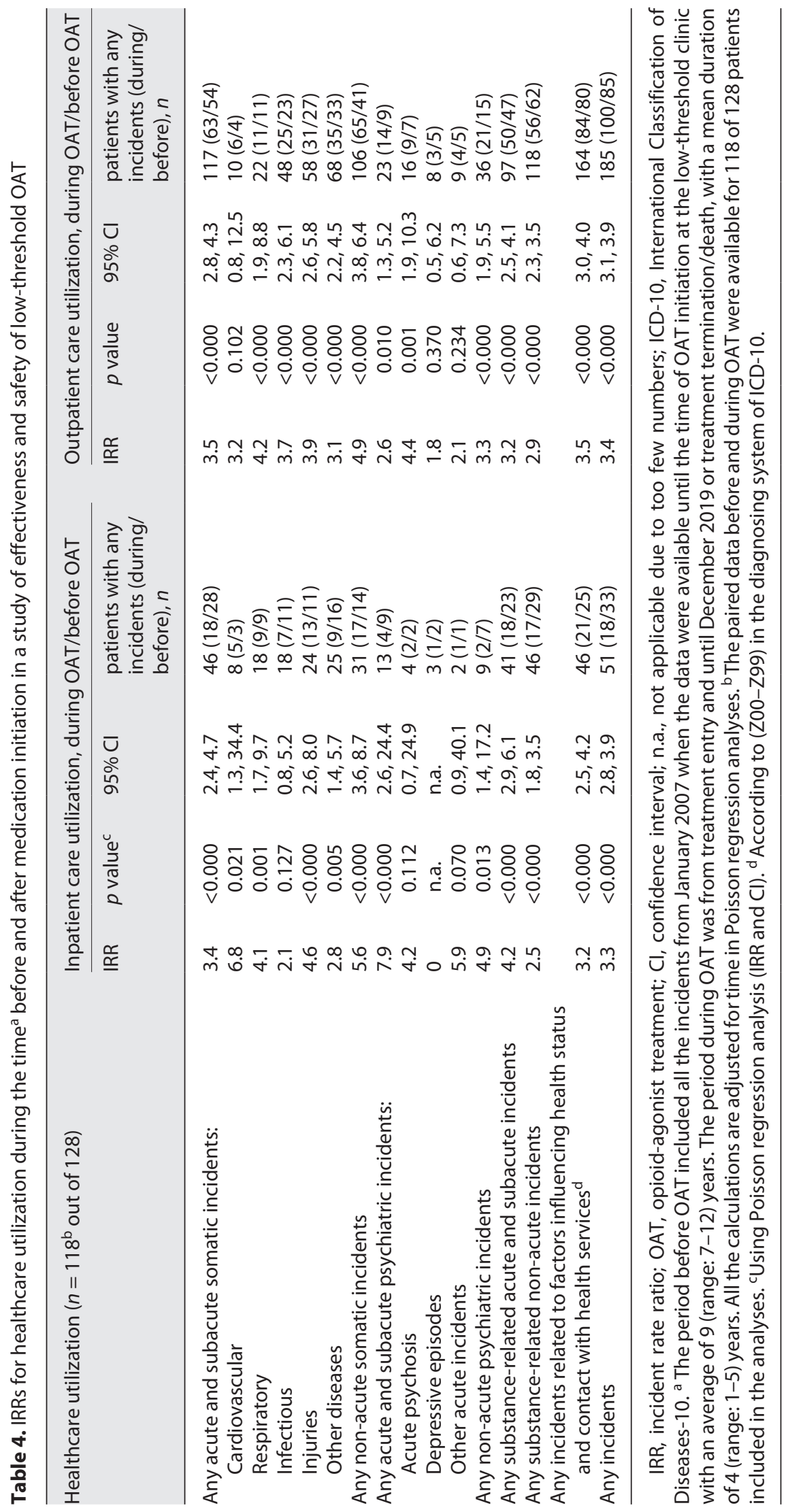




\section{Discussion}

Outpatient treatment initiation without the prerequisites of referral and abstinence from illegal substance use in the B-OAT delivery platform was effective and safe. Retention in treatment and the cumulative rates of dropout, use of illicit opioids, non-fatal overdoses, and death were comparable between the LTC and the NRC. The patients in the LTC were more vulnerable given their social, living, and health status at OAT entrance. Direct comparisons with the NRC were not possible due to the inaccessibility of corresponding OAT entrance data for the same period. The clinical and demographic characteristics of the NRC for the full treatment period, which was longer for the reference group than for the LTC (10 vs. 4 years on average), are therefore presented. Healthcare service use increased substantially during OAT compared to the period prior to OAT, indicating that enrolment to OAT improves access to healthcare in this population.

In this study, the effectiveness of low-threshold OAT was at least as high as that of the standard treatment. Most of the patients continued to receive OAT during the first treatment year and for the full treatment period ( $91 \%$ and $95 \%$, respectively). The voluntary dropout leading to treatment cessation was in line with reports from the NRC and other countries, reflecting one of the challenges in keeping low-adherence patients in treatment [13]. The use of illicit opioids between the LTC and the NRC (7\% vs. $10 \%$, respectively) was also similar. Considering that dropout from OAT increases the risk of relapse to illicit opioid use, overdose, and death $[6,8,9,24,35]$, the importance of low-threshold OAT, which provides easier access and faster re-entrance to OAT, should be further emphasized.

Based on the obtained cumulative rates of non-fatal overdoses and death, our findings suggest that lowthreshold OAT initiation with close clinical observation and frequent follow-ups is as safe as standard treatment. However, the rate of non-fatal overdoses seemed to be higher during the first treatment year than that of the full treatment period, with no significant differences between the Met and Bup groups. This finding points to a smaller subgroup characterized by repeated dropouts, especially during the first year of treatment, and may represent a more hard-to-treat group, which appears inevitable in the NRC as well. There is strong evidence of higher all-cause mortality out of OAT than in OAT [9, 22-25], and a study conducted in 2020 [35] showed that overdose mortality was also lower during OAT than when out of OAT. The risk of overdose death was highest in the week after stop- ping OAT (Met), as well as in the week of Met initiation, but this gradually decreased over time. The researchers also observed elevated overdose mortality rates in the first week after stopping Bup/naloxone [35]. Bup is considered safer than Met $[8,23]$ and is widely prescribed in primary care settings for the treatment of patients with opioid dependence [36]. A Canadian study found that among people who injected drugs, participation in lowthreshold OAT (also among those receiving Met) in primary care settings was significantly associated with improved survival [28]. In contrast, lower OAT coverage and efficacy in Scotland than England/Wales have been mooted as an explanation for the opioid crisis and deaths in Scotland [37].

During this study, 1 death occurred during induction with Met in the LTC. The remaining deaths occurred after the first year following treatment initiation among 4 patients who used Bup before death, 3 of whom were considered to have had drug-related deaths. The findings related to other sedating substances, such as benzodiazepines, in the post-mortem forensic tests may indicate a higher burden of psychiatric comorbidities with more suicidal attempts and risky substance-use behaviours with respect to self-medication. In comparison, somatic diseases were the most frequent causes of death in the NRC, with a mortality study showing $45 \%$ somatic versus $42 \%$ drug-related deaths in the OAT period [38]. Considering the high somatic disease burden in the LTC in our study, our results may be due to the small sample size, which introduced calculation bias. Also, the mean age was higher in the NRC.

The clinical characteristics, including OAT medications and daily doses, and the use of illicit opioids during OAT in the LTC did not differ from those of the general OAT population. However, the study patients represented a more vulnerable subgroup considering their social and living conditions. This also supports the low rate of contact with healthcare services before OAT entrance despite having a high disease burden. Our results demonstrated a significant increase in the utilization of healthcare services during OAT compared to the years prior. Clinically, this was expected since OAT involves extensive and close clinical observation, and patients are encouraged to seek healthcare for medical conditions. High rates of comorbidities exist among patients with opioid dependence [39], and some studies have indicated improvements in the general health status of these patients during OAT [25, 40,41]. Since our study focused on healthcare services use rather than disease burden, we could not determine changes in the incidence, preva- 
lence, severity, or progression of disease during OAT compared to the period before OAT. Our findings of the increased use of healthcare services may indicate that the patients were followed up more closely and referred to the relevant services after OAT entrance as they generally had lower contact with the healthcare system prior to OAT. This may have contributed to the improved health status of the participants.

\section{Limitations and Strengths}

The small sample size of the most marginalized patients compared to the broader national cohort may have influenced our results and is thus a limitation of this study. It was not possible to perform statistical comparisons between the 2 cohorts due to the use of aggregated data in the annual reporting system. We also measured the LTC patient characteristics and some study outcomes at OAT entrance, whereas the data of the NRC were related to the years in treatment. This should be considered a limitation when comparing the 2 cohorts. The presence of confounders and selection bias cannot be disregarded as some results may have been obtained due to differences between the delivery platforms in Bergen and the average national OAT platform. A randomized controlled trial design would limit such biases, but this was not possible in our study as the low-threshold delivery in Bergen was already well established when the study started. A retrospective cohort design was therefore chosen as the second-best option to obtain an impression of the effectiveness and safety of low-threshold OAT initiation in the studied patient group compared with the national OAT cohort. Finally, we could not measure the impact on the disease burden in general among the study participants during OAT compared to the period before OAT due to a lack of baseline data. However, the increased use of healthcare services indicates improvements in the general health status of this population. Notwithstanding the above, this study's strength was the availability of more robust and comprehensive data, which increased the reliability of the findings.

\section{Conclusions}

In this study, low-threshold OAT was found to be at least as effective and safe as the standard OAT in terms of treatment retention, the use of illicit opioids, nonfatal overdoses, and death in people with opioid dependence. The participants' contact with healthcare services increased during the OAT period compared to before
OAT entrance, thus indicating that the most vulnerable groups had access to care, and their health status improved. Met and Bup can be considered safe and effective medications to initiate in an outpatient setting without the prerequisites of a referral or abstinence from substance use. Nevertheless, a cautious dose escalation with close clinical observations should be considered to reduce risks during the induction phase, particularly when using Met [42]. Policymakers and healthcare providers should consider scaling up low-threshold access to OAT through customized delivery platforms to reduce harm and improve the health outcomes of patients with opioid dependence.

\section{Acknowledgments}

We would especially like to thank all the clinical staff at the lowthreshold clinic (M31), Department of Addiction Medicine, Section for Opioid Agonist Treatment, Haukeland University Hospital, for their incredible efforts on providing close care and followup of the participants. We would also like to thank the administrative staff at the department for valuable contribution to data sorting and overview of healthcare utilizations during the project period.

\section{Statement of Ethics}

The study was approved by the Regional Committee for Medical and Health Research Ethics in South-East Norway (approval no. 28499). The committee granted an exemption from the consent requirement due to the use of a hard-to-reach population, the inclusion of death as one of the main outcomes, and a great benefit for medical research with no disadvantages for the participants.

\section{Conflict of Interest Statement}

The authors have declared no conflicts of interest.

\section{Funding Sources}

This work has no funder, and the authors are funded by their respective affiliations.

\section{Author Contributions}

F.C., L.T.F., C.O., and K.A.J. conceived the study and designed it primarily. F.C. is the principal investigator, led statistical analyses in close cooperation with K.A.J. and Ø.A.H., and wrote the first 
draft of the paper. All the authors (F.C., L.T.F., C.O., K.A.J., and $\varnothing$ Ø.A.H.) participated in interpretation of the data, reviewed the manuscript for intellectual content, and approved the final version of the manuscript. The authors confirm their consent for this publication.

\section{Data Availability Statement}

All data generated or analysed during this study are included in this manuscript. No additional data are available due to data protection requirements.

\section{References}

1 Degenhardt L, Whiteford HA, Ferrari AJ, Baxter AJ, Charlson FJ, Hall WD, et al. Global burden of disease attributable to illicit drug use and dependence: findings from the Global Burden of Disease Study 2010. Lancet. 2013;382:1564-74.

2 Krausz RM, Nikoo M, Jang K, Choi F. The North American overdose crisis and the European-American "fentanyl and treatment gap.” Eur Addict Res. 2021;27(4):304-10.

3 Institute for Health Metrics and Evaluation (IHME). GBD results tool. Seattle, WA: IHME, University of Washington; 2021. Available from: http://ghdx.healthdata.org/ gbd-results-tool Accessed 2021 Mar 12.

4 European Monitoring Centre for Drugs and Drug Addiction. European drug report 2013: trends and developments. Lisbon/Luxembourg: European Monitoring Centre for Drugs and Drug Addiction; 2013. Available from: http: //www.emcdda.europa,eu/attachements.cfm/att_213154_EN_ TDAT13001ENNl.pdf.

5 Norwegian Institute for Public Health. Drugrelated deaths in Norway 2020. Norwegian Institute for Public Health; 2021 Jun. Narkotikautløste dødsfall 2020 - FHI.

6 Mattick RP, Kimber J, Breen C, Davoli M. Buprenorphine maintenance versus placebo or methadone maintenance for opioid dependence. Cochrane Database Syst Rev. 2004;3: CD002207.

7 Aas CF, Vold JH, Skurtveit S, Lim AG, Ruths $\mathrm{S}$, Islam $\mathrm{K}$, et al. Health-related quality of life of long-term patients receiving opioid agonist therapy: a nested prospective cohort study in Norway. Subst Abuse Treat Prev Policy. 2020; 15:68.

8 Ma J, Bao YP, Wang RJ, Su MF, Liu MX, Li JQ, et al. Effects of medication-assisted treatment on mortality among opioids users: a systematic review and meta-analysis. Mol Psychiatry. 2019;24:1868-83.

9 Sordo L, Barrio G, Bravo MJ, Indave BI, Degenhardt L, Wiessing L, et al. Mortality risk during and after opioid substitution treatment: systematic review and meta-analysis of cohort studies. BMJ. 2017;357:j1550.

10 Gastberger S, Baumgartner MR, Soyka M, Quednow BB, Hulka LM, Herdener M, et al. Concomitant heroin and cocaine use among opioid-dependent patients during methadone, buprenorphine or morphine opioid agonist therapy. Eur Addict Res. 2019;25:20712.
11 Guillery SPE, Hellweg R, Kronenberg G, Bohr U, Kunte H, Enge S. Quality of life in opioid replacement therapy: a naturalistic cross-sectional comparison of methadone/levomethadone, buprenorphine, and diamorphine patients. Eur Addict Res. 2021;30:1-10.

12 Volkow ND, Frieden TR, Hyde PS, Cha SS. Medication-assisted therapies - tackling the opioid-overdose epidemic. N Engl J Med. 2014;370:2063-6.

13 Kourounis G, Richards BD, Kyprianou E, Symeonidou E, Malliori MM, Samartzis L. Opioid substitution therapy: lowering the treatment thresholds. Drug Alcohol Depend. 2016;161:1-8.

14 Adamson SJ, Sellman JD. The pattern of intravenous drug use and associated criminal activity in patients on a methadone treatment waiting list. Drug Alcohol Rev. 1998;17:15966.

15 Gjersing L, Bretteville-Jensen AL. Is opioid substitution treatment beneficial if injecting behaviour continues? Drug Alcohol Depend. 2013;133:121-6.

16 Bach P, Milloy MJ, Nguyen P, Koehn J, Guillemi S, Kerr T, et al. Gender differences in access to methadone maintenance therapy in a Canadian setting. Drug Alcohol Rev. 2015;34: 503-7.

17 Peles E, Schreiber S, Adelson M. 15-Year survival and retention of patients in a general hospital-affiliated methadone maintenance treatment (MMT) center in Israel. Drug Alcohol Depend. 2010;107:141-8.

18 Perreault M, Julien D, White ND, Rabouin D, Lauzon P, Milton D. Psychological predictors of retention in a low-threshold methadone maintenance treatment for opioid addicts: a 1-year follow-up study. Subst Use Misuse. 2015;50:24-31.

19 Reimer J, Vogelmann T, Trümper D, Scherbaum N. Impact of buprenorphine dosage on the occurrence of relapses in patients with opioid dependence. Eur Addict Res. 2020;26:77-84.

20 Kwan TH, Wong NS, Lee SS. Participation dynamics of a cohort of drug users in a lowthreshold methadone treatment programme. Harm Reduct J. 2015;12:30.

21 Buster MC, van Brussel GH, van den Brink W. An increase in overdose mortality during the first 2 weeks after entering or re-entering methadone treatment in Amsterdam. Addiction. 2002;97:993-1001.
22 Degenhardt L, Randall D, Hall W, Law M, Butler T, Burns L. Mortality among clients of a state-wide opioid pharmacotherapy program over 20 years: risk factors and lives saved. Drug Alcohol Depend. 2009;105:9-15.

23 Kimber J, Larney S, Hickman M, Randall D, Degenhardt L. Mortality risk of opioid substitution therapy with methadone versus buprenorphine: a retrospective cohort study. Lancet Psychiatry. 2015;2:901-8.

24 Clausen T, Anchersen K, Waal H. Mortality prior to, during and after opioid maintenance treatment (OMT): a national prospective cross-registry study. Drug Alcohol Depend. 2008;94:151-7.

25 Skeie I, Brekke M, Gossop M, Lindbaek M, Reinertsen E, Thoresen M, et al. Changes in somatic disease incidents during opioid maintenance treatment: results from a Norwegian cohort study. BMJ Open. 2011;1: e000130.

26 Gervasoni JP, Balthasar H, Huissoud T, Jeannin A, Dubois-Arber F. A high proportion of users of low-threshold facilities with needle exchange programmes in Switzerland are currently on methadone treatment: implications for new approaches in harm reduction and care. Int J Drug Policy. 2012;23:33-6.

27 Ahmad K, Hayashi K, Nguyen P, Dobrer S, Kerr T, Schütz CG, et al. Effect of low-threshold methadone maintenance therapy for people who inject drugs on HIV incidence in Vancouver, BC, Canada: an observational cohort study. Lancet HIV. 2015;2:e445-50.

28 Nolan S, Hayashi K, Milloy MJ, Kerr T, Dong $\mathrm{H}$, Lima VD, et al. The impact of low-threshold methadone maintenance treatment on mortality in a Canadian setting. Drug Alcohol Depend. 2015;156:57-61.

29 Nordt C, Stohler R. Low-threshold methadone treatment, heroin price, police activity and incidence of heroin use: the Zurich experience. Int J Drug Policy. 2009;20:497-501.

30 Strike C, Millson M, Hopkins S, Smith C. What is low threshold methadone maintenance treatment? Int J Drug Policy. 2013;24: e51-6.

31 Henriksen K, Jacobsen JA, Henriksen EM, Gomes L, Waal H, Krajci P. The lasso program in Oslo: harm reduction using buprenrophine-naloxone (subxone ${ }^{\varpi}$ ) in a low threshold setting. Eur Addict Res. 2018;24:286-92.

32 Norwegian national guidelines for medication assisted rehabilitation in opioid addiction. Norwegian Health Directorate; 2010. IS1701. 
33 Fadnes LT, Aas CF, Vold JH, Ohldieck C, Leiva RA, Chalabianloo F, et al. Integrated treatment of hepatitis $C$ virus infection among people who inject drugs: study protocol for a randomised controlled trial (INTRO-HCV). BMC Infect Dis. 2019;19:943.

34 Lobmaier P, Skeie I, Lillevold P, Waal H, Bussesund K, Clausen T. National status report on opioid agonist treatment in Norway. SERAF; 2020. Issued 2020 Jun 29.

35 Pearce LA, Min JE, Piske M, Zhou H, Homayra F, Slaunwhite A, et al. Opioid agonist treatment and risk of mortality during opioid overdose public health emergency: population based retrospective cohort study. BMJ. 2020;368:m772.
36 Dupouy J, Maumus-Robert S, Mansiaux Y, Pariente A, Lapeyre-Mestre M. Primary care of opioid use disorder: the end of "the French model"? Eur Addict Res. 2020;26:346-54.

37 van Amsterdam J, van den Brink W, Pierce M. Explaining the differences in opioid overdose deaths between Scotland and England/Wales: implications for European opioid policies. Eur Addict Res. 2021;7:1-14.

38 Bech AB, Clausen T, Waal H, Šaltyte Benth J, Skeie I. Mortality and causes of death among patients with opioid use disorder receiving opioid agonist treatment: a national register study. BMC Health Serv Res. 2019;19:440.

39 Peacock A, Leung J, Larney S, Colledge S, Hickman M, Rehm J, et al. Global statistics on alcohol, tobacco and illicit drug use: 2017 status report. Addiction. 2018;113:1905-26.

40 Darke S, Ross J, Teesson M. The Australian treatment outcome study (ATOS): what have we learnt about treatment for heroin dependence? Drug Alcohol Rev. 2007;26:49-54.

41 Gossop M, Stewart D, Browne N, Marsden J. Methadone treatment for opiate dependent patients in general practice and specialist clinic settings: outcomes at 2-year follow-up. J Subst Abuse Treat. 2003;24:313-21.

42 Baxter LE, Campbell A, Deshields M, Levounis P, Martin JA, McNicholas L, et al. Safe methadone induction and stabilization: report of an expert panel. J Addict Med. 2013;7: $377-86$. 